\title{
Multi-modality imaging of post-myocardial infarction ventricular septal defect associated to basal inferoseptal pseudoaneurysm
}

\author{
Giovanni Barbati $^{1}$ (D) $\cdot$ Giovanna Erente $^{1} \cdot$ Paolo Magagna $^{2} \cdot$ Luca Spigolon $^{3} \cdot$ Francesco Caprioglio $^{1}$
}

Received: 16 May 2021 / Revised: 24 June 2021 / Accepted: 3 September 2021

(C) Japanese Society of Echocardiography 2021

An 84 year-old man, admitted in Intensive Cardiac Care Unit during the COVID-19 pandemic for late presenting inferoposterior myocardial infarction (MI), showed an holosystolic murmur along left sternal border consistent with suspected intracardiac shunt at focused cardiac ultrasound. Coronary angiography detected a thrombotic occlusion of distal right coronary artery and ventriculography revealed a large basal inferoseptal aneurysm associated to left-to-right shunt (Fig. 1a). After consulting heart team, it was decided to treat right coronary occlusion with angioplasty and stent implantation, postponing the defect treatment in the next days. Transthoracic echocardiography (TTE) clearly displayed that it was a huge pseudoaneurysm with a wide neck, associated to a ventricular septal defect with a significant left-to-right shunt (Fig. 1b and 1c, Video 1 of the electronic supplementary material), calculated QP/QS of 1,9 and transventricular gradient of $72 \mathrm{mmHg}$ (Fig. 1d). 3D TTE, using a photorealistic rendering, gave the possibility to navigate into the lesions with an en-face perspective (Fig. 1e,f) and to reconstruct their dimensions with a multiplanar approach (Video 2 of electronic supplementary material). The neck of the pseudoaneurysm resulted $21 \times 22 \mathrm{~mm}$, whilst the ventricular septal defect measured $8 \times 10 \mathrm{~mm}$. To further best characterize these lesions to attempt a percutaneous closure, we performed a computed tomography (Fig. 1g), from which a 3D plastic model was obtained (Fig. 1h). After 18 days from percutaneous coronary intervention, the patient underwent percutaneous closure of defects. Despite treatment, post-operative course was complicated by cardiogenic shock due to right heart failure, consequence of right ventricle involvement in late presenting inferoposterior myocardial infarction and of left-to-right shunt, which eventually led to patient demise for multiorgan failure after eight days from intervention.

During COVID-19 pandemic we are assisting to an increase of post-MI mechanical complications [1]. Ventricular septal defect associated to left ventricular pseudoaneurysm represents a rare and life-threatening mechanical complication of late presenting MI [2,3]. Few cases are described in literature and the optimal treatment is debating. Using multi-modality imaging is mandatory to optimally define the anatomy before attempting a percutaneous or surgical treatment. 3D printing models, particularly, can be very attracting and useful for deep understanding of morphology of such complex cardiac defects.

Giovanni Barbati

giovanni.barbati@aulss8.veneto.it

1 Department of Cardiology, San Bortolo Hospital, Viale Rodolfi 37, 36100 Vicenza, Italy

2 Department of Cardiac Surgery, San Bortolo Hospital, Viale Rodolfi 37, 36100 Vicenza, Italy

3 Department of Radiology, San Bortolo Hospital, Viale Rodolfi 37, 36100 Vicenza, Italy 


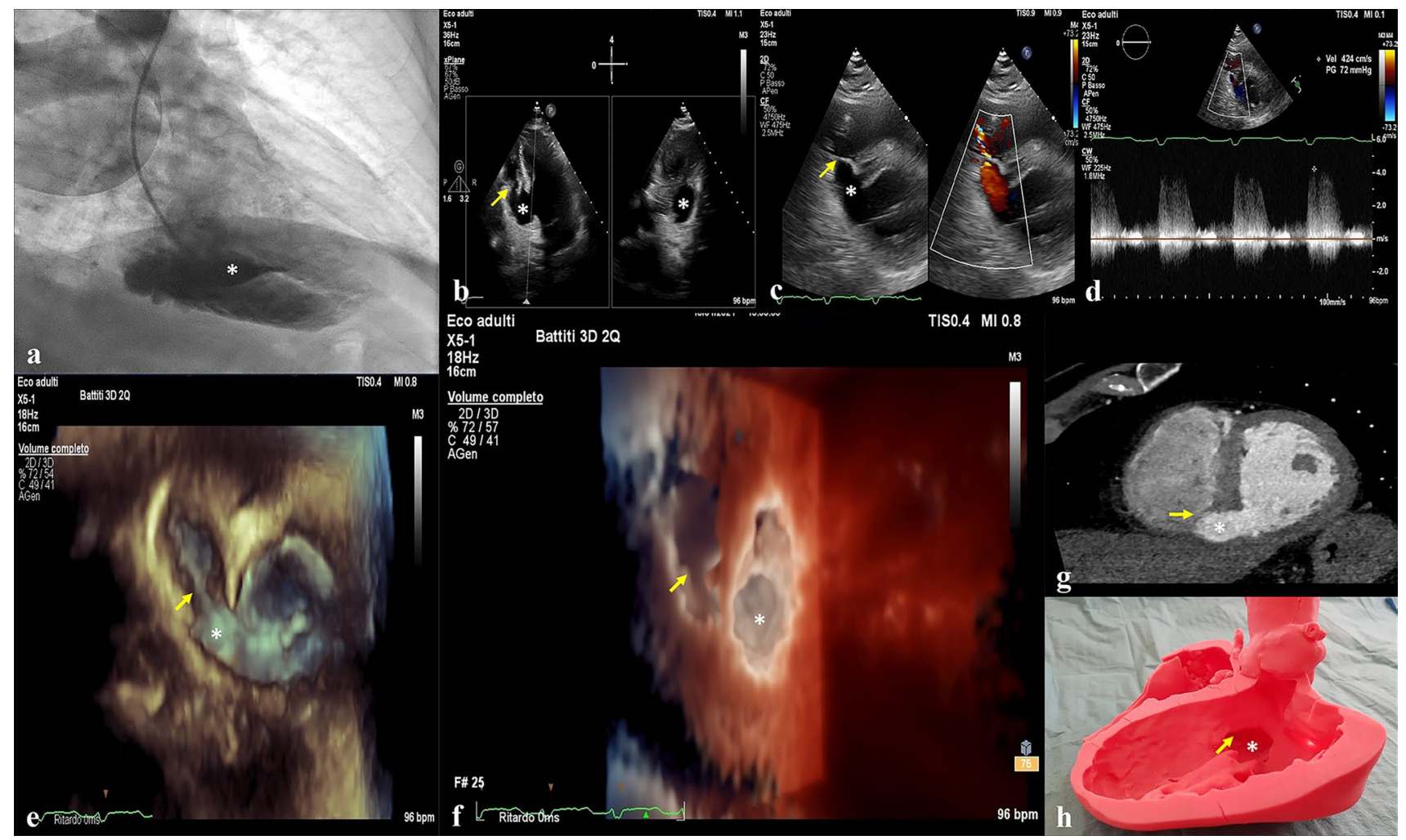

Fig. 1 Ventriculography and TTE displaying the basal inferoseptal pseudoaneurysm (a and b); TTE, with color and CW Doppler, demonstrating the presence of ventricular septal defect with a significant left-to-right shunt (c and d); 3D TTE, with photorealistic rendering,

Supplementary Information The online version contains supplementary material available at https://doi.org/10.1007/s12574-021-00551-8.

Author contributions All authors contributed to the case report conception. Material preparation of images was performed by GB. The first draft of the manuscript was written by GB and all authors commented on previous versions of the manuscript. All authors read and approved the final manuscript.

\section{Declarations}

Conflict of interest Giovanni Barbati, Giovanna Erente, Paolo Magagna, Luca Spigolon and Francesco Caprioglio declare that they have no conflict of interest.

\section{References}

1. Kitahara S, Fujino M, Honda S, Yasuhide Asaumi Yu, Kataoka FO, et al. COVID-19 pandemic is associated with mechanical giving the possibility to navigate into the lesions (e and f); computed tomography image of defects (g) and 3D plastic model (h) obtained to best characterize them; ventricular septal defect (yellow arrow); pseudoaneurysm (white asterisk)

complications in patients with ST-elevation myocardial infarction. Open Heart. 2021;8: e001497. https://doi.org/10.1136/openh rt-2020-0011497.

2. Inoue T, Hashimoto K, Bando Ko, Yoshitake M. Left ventricular pseudo-false aneurysm perforating into the right ventricle. Interact Cardiovasc Thorac Surg. 2015;21:137-9.

3. Agarwal C, Goel S, Jacobi A, Love B, Sanz J. CT imaging of postmyocardial infarction ventricular septal defect with a contained rupture/pseudoaneurysm. Indian Heart J. 2015;67:S107-109.

Publisher's Note Springer Nature remains neutral with regard to jurisdictional claims in published maps and institutional affiliations. 